PROCEEDINGS OF THE

AMERICAN MATHEMATICAL SOCIETY

Volume 135, Number 10, October 2007, Pages 3039-3040

S 0002-9939(07)08797-7

Article electronically published on February 6, 2007

\title{
A SMOOTH INVARIANT NOT A SMOOTH FUNCTION OF THE INVARIANT POLYNOMIALS
}

\author{
LARRY BATES
}

(Communicated by Martin Lorenz)

\begin{abstract}
An explicit example is given of a smooth function invariant under a linear group action that is not a smooth function of the invariant polynomials.
\end{abstract}

A deep theorem of Schwarz [1] asserts that a smooth function invariant under the linear action of a compact Lie group is a smooth function of the invariant polynomials. A simple example is given to show that this does not extend to noncompact groups, at least if the action is not proper.

Consider the motion of a free particle in the plane $\mathbb{R}^{2}$, with Cartesian coordinates $x_{1}, x_{2}$. This is modelled by the Hamiltonian system with the phase space $P=$ $T^{*} \mathbb{R}^{2}$ and the Hamiltonian $h=\frac{1}{2}\left(v_{1}^{2}+v_{2}^{2}\right)$. The Hamiltonian flow is the linear action $\left(t,\left(x_{1}, x_{2}, v_{1}, v_{2}\right)\right) \rightarrow\left(x_{1}+t v_{1}, x_{2}+t v_{2}, v_{1}, v_{2}\right)$, which is two copies of the representation on $\mathbb{R}^{2}$ given by the matrix $t \rightarrow\left(\begin{array}{ll}1 & 0 \\ t & 1\end{array}\right)$. Observe that not only are $v_{1}, v_{2}$ and $j:=x_{1} v_{2}-x_{2} v_{1}$ constants of motion, but are a basis for the invariant polynomials by the following lemma.

Lemma. Suppose that $f$ is a polynomial on $P$ that is invariant under the Hamiltonian flow. Then $f$ is a polynomial in the invariant polynomials $v_{1}, v_{2}, j$.

Proof. Let $f$ be invariant under the flow. Then expanding by Taylor's theorem and denoting by $\partial_{k}$ differentiation with respect to $x_{k}$

$$
\begin{aligned}
f\left(x_{1}, x_{2}, v_{1}, v_{2}\right) & =f\left(x_{1}+t v_{1}, x_{2}+t v_{2}, v_{1}, v_{2}\right) \\
& =f\left(x_{1}, x_{2}, v_{1}, v_{2}\right)+t\left(v_{1} \partial_{1}+v_{2} \partial_{2}\right) f\left(x_{1}, x_{2}, v_{1}, v_{2}\right)+t^{2}(\cdots) .
\end{aligned}
$$

Since $v_{1}, v_{2}$ are irreducible elements and distinct, we may conclude that

$$
v_{1} \partial_{1} f+v_{2} \partial_{2} f=0 .
$$

Therefore, since we are in a unique factorization domain, it follows that

$$
\partial_{1} f=-v_{2} p, \quad \partial_{2} f=v_{1} p
$$

for some polynomial $p$. The key observation to make is that the operator $x_{1} \partial_{1}+x_{2} \partial_{2}$ acts as scalar multiplication by $n$ on a homogeneous polynomial in $x_{1}, x_{2}$ of degree

Received by the editors May 8, 2006.

2000 Mathematics Subject Classification. Primary 14L24, 16W22.

Key words and phrases. Geometric invariant theory.

Partially supported by NSERC.

(C)2007 American Mathematical Society Reverts to public domain 28 years from publication 
$n$. Now write

$$
f=\sum_{n \geq 0} f_{n}, \quad p=\sum_{n \geq 0} p_{n}
$$

as homogeneous expansions in $x_{1}, x_{2}$ over $\mathbb{R}\left[v_{1}, v_{2}\right]$. Equation (1) implies that

$$
\left(x_{1} \partial_{1}+x_{2} \partial_{2}\right) f=\left(x_{2} v_{1}-x_{1} v_{2}\right) p
$$

or, expanding, that

$$
\left(x_{1} \partial_{1}+x_{2} \partial_{2}\right) \sum_{n \geq 0} f_{n}=\sum_{n \geq 1} n f_{n}=\sum_{n \geq 0} p_{n}\left(x_{2} v_{1}-x_{1} v_{2}\right) .
$$

Comparing coefficients yields

$$
f_{n}=\frac{p_{n-1}}{n}\left(x_{2} v_{1}-x_{1} v_{2}\right)
$$

so that

$$
f=f_{0}+\left(x_{2} v_{1}-x_{1} v_{2}\right)\left(\frac{p_{0}}{1}+\frac{p_{1}}{2}+\cdots\right) .
$$

Since $f, f_{0}$ and $x_{2} v_{1}-x_{1} v_{2}$ are all invariants, it follows that

$$
\left(\frac{p_{0}}{1}+\frac{p_{1}}{2}+\cdots\right)
$$

is as well. However, in $x_{1}$ and $x_{2}$ it is of degree one less. Hence, by induction, $f$ is a polynomial in $v_{1}, v_{2}$ and $j$.

Now consider the function $f$ on the phase space $P$ defined by

$$
f\left(x_{1}, x_{2}, v_{1}, v_{2}\right)=\frac{e^{-1 / j^{2}}}{v_{1}^{2}+v_{2}^{2}}
$$

and $f=0$ when $j=0$. Since $j^{2} \leq\left(x_{1}^{2}+x_{2}^{2}\right)\left(v_{1}^{2}+v_{2}^{2}\right), f$ is smooth on phase space. However, $f$, thought of as a function on the image of the invariants map $J:\left(x_{1}, x_{2}, v_{1}, v_{2}\right) \rightarrow\left(v_{1}, v_{2}, j\right)$, is not a smooth function as it has no continuous extension to any neighbourhood of the origin. Note that the image of $J$ is all of $\mathbb{R}^{3}$ with the $j$ axis removed and the origin stuck back in. It is the peculiar form of the image of $J$ that explains why it is possible for the pullback $J^{*} f$ to be smooth.

It is a pleasure to thank H. Farahat for his patient explanations showing me what was really going on in the lemma.

\section{REFERENCES}

[1] G. Schwarz. Smooth functions invariant under the action of a compact Lie group. Topology, 14:63-68, 1975. MR0370643(51:6870)

Department of Mathematics and Statistics, University of Calgary, Calgary, AlBERTA, T2N 1N4 CANADA 